\title{
Unusual endophthalmitis with macular infarction
}

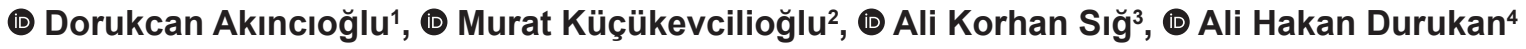 \\ ${ }^{1}$ Antalya Ataturk State Hospital, Clinic of Ophthalmology, Antalya, Turkey \\ 2University of Health Sciences Turkey, Gülhane Training and Research Hospital, Clinic of Ophthalmology, Ankara, Turkey \\ 3Hacettepe University Faculty of Medicine, Department of Medical Microbiology, Ankara, Turkey \\ 4University of Health Sciences Turkey, Gülhane Faculty of Medicine, Department of Ophthalmology, Ankara, Turkey
}

Date submitted:

19.05.2020

Date accepted:

25.06.2020

Online publication date:

15.03.2021

\section{Corresponding Author:}

Dorukcan Akıncıoğlu MD PhD, Antalya

Ataturk State Hospital, Clinic of

Ophthalmology, Antalya, Turkey

dr.dorukcan@yahoo.com

ORCID:

orcid.org/0000-0001-6409-9802

Keywords: Endophthalmitis, infarction, Klebsiella, macula

\begin{abstract}
Macular infarction due to intraocular aminoglycoside injection is well known. Recently, few atypical organisms have been reported to cause macular infarction after endophthalmitis. Our case is a 69-year-old female patient who presented with redness and discharge in the right eye. She had had intraocular ranibizumab injection for diabetic macular edema two days ago. The vision was counting fingers at one meter without a clear view of the fundus. The patient underwent vitreous tap, and intraocular injection of vancomycin and ceftazidime was performed. The vitreous culture was positive for Klebsiella pneumoniae. Two days later, fundus visibility was better. However, the vision was counting fingers at 50 centimeters, and macula was pale. Therefore, we planned pars plana vitrectomy. Klebsiella pneumonaie is generally considered to be a cause of endogenous endophthalmitis, especially in patients with liver abscess or fulminant pneumonia. We herein wanted to report the first case of Klebsiella pneumonaie endophthalmitis with no endogenous focus resulting in macular infarction.
\end{abstract}

\section{Introduction}

Intravitreal injections (IVI) have become the mainstay therapy for various retinal diseases following the demonstration of beneficial roles by multicenter clinical trials. On the other hand, endophthalmitis is one of the most feared complications. The incidence of endophthalmitis following IVI varies from 0 to $0.092 \%(1,2)$. It is crucial to identify the causative organism due to therapeutic challenges in unknown etiologies. A detailed history is essential to narrow down the list of organisms. Macular infarction following intravitreal amikacin injections is well known (3). Enterococcus faecalis and Staphylococcus epidermidis are previously reported as the causative agents of macular infarction in endophthalmitis as well (4). Klebsiella pneumoniae is a causative organism of endogenous endophthalmitis, especially in diabetic patients with accompanying hepatic or renal abscess $(5,6)$. In this report, we present the first case with macular infarction secondary to exogenous Klebsiella pneumoniae endophthalmitis with no underlying systemic pathology.

\section{Case Presentation}

A 69-year-old white female patient presented with pain, redness, blurred vision, and discharge in the right eye two days after intravitreal ranibizumab injection. She was diabetic, and she had a diagnosis of proliferative diabetic retinopathy with diabetic macular edema before the IVI. At the initial examination, her bestcorrected visual acuity was 20/1250 in the right eye and 20/25 in the left eye. On slit-lamp examination, hazy cornea and mixed cells with fibrin in the anterior chamber were observed. Vitreous was hazy with a severe cellular response. The afferent pupillary defect was visible in the involved right eye. Ocular motility was normal though the pain was worse with eye movements. Poor red reflex was observed, but B-scan ultrasonography revealed 
an attached retina. A diagnosis of acute endophthalmitis was made with clinical findings of light sensitivity, ocular paindecreased vision, and severe cellular response in vitreous. The patient underwent vitreous and anterior chamber tap followed by the injection of vancomycin ( $1 \mathrm{mg} / 0.1 \mathrm{cc}$ ) and ceftazidime (2.25 mg/0.1 cc). The patient was admitted and started on topical fortified vancomycin $(50 \mathrm{mg} / \mathrm{mL})$, ceftazidime $(100 \mathrm{mg} /$ $\mathrm{mL}$ ), and prednisolone acetate every hour as well as peroral moxifloxacin (400 mg) once daily. On the following day, there was no clinical improvement, and vision further decreased to $20 / 25000$. We proceeded with vitrectomy. During the surgery, a pale macula with multiple retinal hemorrhages was observed (Figure 1). Following surgery, fluorescein angiography (FA) revealed macular ischemia, and optical coherence tomography showed subretinal fluid (Figure 2).

Culture of vitreous fluid was obtained, and following proper inoculation and two days of incubation, the growth of Gramnegative bacilli was observed. Conventional biochemical analysis and matrix-assisted laser desorption/ionizationtime of flight mass spectrometry (Bruker, MA, USA) revealed the identification as Klebsiella pneumoniae. Antimicrobial susceptibility tests were applied in accordance with the European Committee on Antimicrobial Susceptibility Testing criteria (Figure 1D) (7). The species showed susceptibility to cefepime, cefotaxime, ceftriaxone, ceftazidime, ciprofloxacin, gentamicin, imipenem, meropenem, and netilmicin; intermediate susceptibility to piperacillin - tazobactam; resistance to amoxicillin - clavulanate and ampicillin - sulbactam. Klebsiella pneumoniae endophthalmitis is a commonly endogenous and delayed diagnosis of endogenous endophthalmitis. The endogenous microorganism can lead to not only visual loss but also an increased risk of mortality. Infectious disease and internal medicine consultations were requested. Blood culture, blood

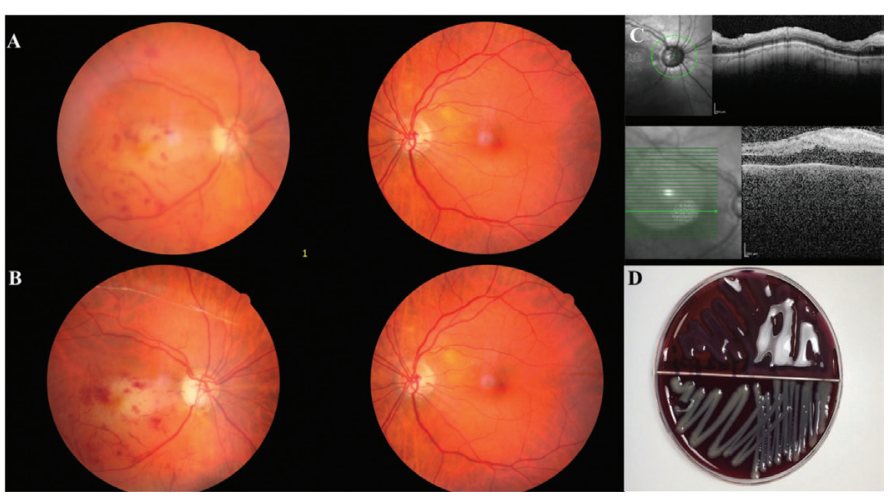

Figure 1. Before pars plana vitrectomy retinal images were not clear. These images are taken following vitrectomy. A) Same day, following pars plana vitrectomy. The pale macula and retinal hemorrhages are seen. B) One week after following pars plana vitrectomy. Macular ischemia is advanced. C) The retinal nerve fiber layer has not been affected yet. Subretinal fluid and retinal edema are seen due to vascular ischemia and inflammation. Macular thickness is $448 \mu \mathrm{m} \mathrm{D)} \mathrm{Klebsiella}$ pneumonaie subculture after $24^{\text {th }}$ incubation analysis, and related imaging modalities (thoraco-abdominal computed tomography and abdominal ultrasonography) for any abscess revealed no endogenous focus of infection.

At her 1-month follow-up visit, the vision was still poor. However, subretinal fluid resolved without any intervention (Figure 2). At her final visit (four months after diagnosis), the vision was unchanged with the resolution of anterior chamber reaction, and a few preretinal macular hemorrhages were observed. Figure $2 \mathrm{G}$ and Figure $2 \mathrm{H}$ show retinal and vascular change by time.

\section{Discussion}

Intravitreal aminoglycoside toxicity is generally acknowledged as a predisposing factor for macular infarction. Therefore, we substituted ceftazidime for aminoglycosides due to potential hazards in the treatment of bacterial endophthalmitis $(7,8)$. Ceftazidime is a third-generation cephalosporin with particularly good coverage of Gram-negative bacteria with low toxicity in comparison with aminoglycosides. Intravitreal vancomycin is considered to be safe if injected at doses of $1 \mathrm{gram}$ and intervals of 48 hours (9). On the other hand, macular infarction, possible retinal toxicity of aminoglycosides, may also be caused by infective agents like Enterococcus faecalis and Staphylococcus epidermidis $(3,4)$.

Klebsiella pneumoniae is commonly the causative agent of endogenous endophthalmitis. Endogenous endophthalmitis, comprising $2 \%$ to $15 \%$ of all endophthalmitis, is a rare but devastating ocular infection when an organism crosses

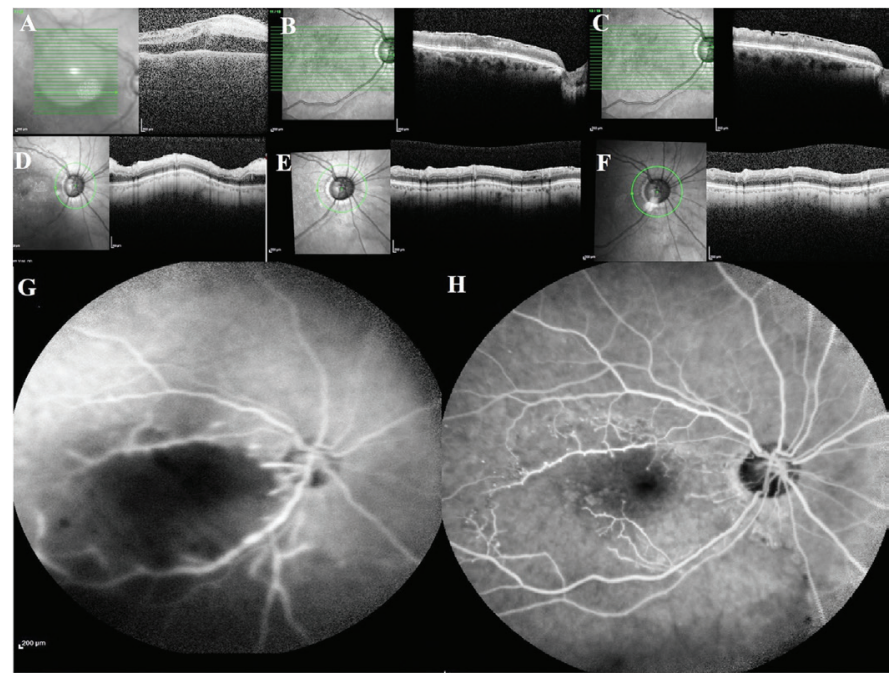

Figure 2. Macular thickness and retinal nerve fiber layer thickness by time. A) Image at the time of diagnosis following pars plana vitrectomy. Macular thickness is $448 \mu \mathrm{m}$. B) One year later follow-up exam, the macular thickness is $185 \mu \mathrm{m}$. C) Three-year follow-up exam, the macular thickness is $135 \mu \mathrm{m}$. D) Optic disc image at the time of diagnosis. E) One-year follow-up exam. F) Three-year follow-up exam. G) Macular ischemia at the time of diagnosis following pars plana vitrectomy. $\mathrm{H}$ ) Areas of capillary drop out one year later 
through the blood-ocular barrier. These patients are usually immunocompromised related to their systemic problems like diabetes mellitus, renal insufficiency, and malignancy. A major source for Klebsiella pneumoniae endogenous endophthalmitis (KPEE) is a liver abscess with septic metastasis to the eye (10). Clinical manifestation for KPEE is not unique though accompanying previous systemic health problems with or without Klebsiella pneumoniae infection should remind us a possible KPEE. Our case had only diabetes mellitus as a possible predisposing factor, and serotype $\mathrm{K} 1$ and $\mathrm{K} 2$ are reported with increased pathogenicity in diabetic patients employing their virulence factors (11). Unfortunately, visual prognosis in KPEE is very poor though macular infarction is not reported previously. Probably macular infarction was the main reason for this poor prognosis and missed because FA is not routinely performed in endophthalmitis cases.

\section{Conclusion}

Our case is unique with no possible endogenous focus of the causative agent and unusual presentation of Klebsiella pneumoniae endophthalmitis with macular infarction. In this regard, endophthalmitis with macular infarction should remind us Klebsiella pneumoniae as causative agents, even predisposing systemic factors are lacking.

\section{Ethics}

Informed Consent: The patient gave informed consent to publish the case report without revealing her identity.

Peer-review: Externally peer-reviewed.

Surgical and Medical Practices: M.K., A.H.D., Concept: D.A., Design: D.A., A.H.D., Data Collection or Processing: D.A., A.K.S., Analysis or Interpretation: D.A., M.K., A.K.S., A.H.D., Literature Search: D.A., M.K., A.K.S., Writing: D.A., A.K.S.

Conflict of Interest: No conflict of interest was declared by the authors.

Financial Disclosure: The authors declared that this study received no financial support.

\section{References}

1. Brynskov $T$, Kemp $H$, Sorensen TL. No cases of endophthalmitis after 20,293 intravitreal injections in an operating room setting. Retina. 2014;34:951-957.

2. Day S, Acquah K, Mruthyunjaya P, Grossman DS, Lee PP, Sloan FA. Ocular complications after anti-vascular endothelial growth factor therapy in Medicare patients with age-related macular degeneration. Am J Ophthalmol. 2011;152:266-272.

3. Galloway G, Ramsay A, Jordan K, Vivian A. Macular infarction after intravitreal amikacin: mounting evidence against amikacin. Br J Ophthalmol. 2002;86:359-360.

4. Kunjukunju N, Gonzales CR, Rodden WS. Macular infarction secondary to Staphylococcus epidermidis infection. Retin Cases Brief Rep. 2012;6:56-59.

5. C. Yang CS, Tsai HY, Sung CS, Lin KH, Lee FL, Hsu WM. Endogenous Klebsiella endophthalmitis associated with pyogenic liver abscess. Ophthalmology. 2007;114:876880.

6. Liu YC, Cheng DL, Lin CL. Klebsiella pneumoniae liver abscess associated with septic endophthalmitis. Arch Intern Med. 1986;146:1913-1916.

7. Mannerström M, Zorn-Kruppa M, Diehl H, et al. Evaluation of the cytotoxicity of selected systemic and intravitreally dosed drugs in the cultures of human retinal pigment epithelial cell line and of pig primary retinal pigment epithelial cells. Toxicol In Vitro. 2002;16:193-200.

8. Campochiaro PA, Lim Jl. Aminoglycoside toxicity in the treatment of endophthalmitis. The Aminoglycoside Toxicity Study Group. Arch Ophthalmol. 1994;112:48-53.

9. Libert J. Toxicity of intravitreal injections of antibiotics and antivirals. Bull Soc Belge Ophthalmol. 2007;304:89-97.

10. Chen KJ, Hwang YS, Wang NK, Chao AN. Endogenous Klebsiella pneumoniae endophthalmitis with renal abscess: report of two cases. Int J Infect Dis. 2010;14:429-432.

11. Sachdev DD, Yin MT, Horowitz JD, Mukkamala SK, Lee SE, Ratner AJ. Klebsiella pneumoniae K1 liver abscess and septic endophthalmitis in a U.S. resident. J Clin Microbiol. 2013;51:1049-1051. 